\title{
TENDÊNCIAS DA MORTALIDADE EM MULHERES BRASILEIRAS NO CLIMATÉRIO
}

\section{MORTALITY TRENDS AMONG BRAZILIAN WOMEN IN THE CLIMACTERIC}

\author{
Ana Carolina Basso Schmitt* \\ Maria Regina Alves Cardoso** \\ José Mendes Aldrighi***
}

Schmitt ACB, Cardoso MRA, Aldrighi JM. Tendências da mortalidade em mulheres brasileiras no climatério. Rev Bras Crescimento Desenvol Hum. 2008; 18(1): 11-15.

\begin{abstract}
Resumo: Há poucos estudos sobre mortalidade feminina durante o climatério, em especial no Brasil. O objetivo deste estudo foi analisar a tendência de mortalidade em mulheres de 35 a 64 anos no Brasil nos últimos anos. Para tanto, foram coletados os dados de mortalidade do Sistema de Informações de Mortalidade do Datasus, Ministério da Saúde, para o período de 1979 a 2004. Para análise, foram calculados os coeficientes específicos de mortalidade por idade e causa para os dez capítulos da Classificação Internacional de Doenças mais freqüentes como causa de morte por década da faixa etária do climatério, nas regiões do Brasil. No Brasil, três capítulos da Classificação Internacional de Doenças predominaram: doenças do aparelho circulatório; neoplasias e causas mal definidas. As regiões Sudeste, Sul e Centro-Oeste acompanharam o mesmo padrão do país, em relação à posição das três primeiras causas de morte, contudo, as doenças do aparelho circulatório e as causas mal definidas decresceram e as neoplasias aumentaram. $\mathrm{Na}$ região Norte, apesar das mesmas causas apresentarem coeficientes próximos, as doenças circulatórias prevaleceram na maior parte do período estudado, mas as causas mal definidas foram mais freqüentes que as neoplasias. Já no Nordeste, as principais causas foram as mal definidas, embora tenham decrescido de 1979 a 2004. As doenças do aparelho circulatório e neoplasias ocuparam a segunda e terceira posições, respectivamente, e aumentaram no período de estudo.
\end{abstract}

Palavras-chave: Mortalidade; saúde da mulher; epidemiologia.

\section{INTRODUÇÃO}

A expectativa de vida da população geral brasileira tem aumentado nos últimos anos, consoante o World Health Organization Statistical Information System, alcançando 74 anos para as mulheres e 72 anos para os homens no ano de $2004^{1}$.

Apesar disso, diversas mulheres adoecem e morrem antes desta idade e nota-se uma lacuna de estudos no tocante à mortalidade de mu- lheres no climatério.

O climatério, fase da vida da mulher que marca a transição do período reprodutivo para o não reprodutivo com início aos 35 e término aos 65 $\operatorname{anos}^{2}$ caracteriza-se por uma redução progressiva dos esteróides sexuais ovarianos, que redunda em sintomas desconfortáveis e graves doenças ${ }^{3}$.

Entre os sintomas desconfortáveis, incluem-se, entre tantos: as ondas de calor, a insônia, a secura da vagina e entre as doenças: a depressão, a osteoporose, a obesidade, as dislipidemias

\footnotetext{
Departamento de Fisioterapia - UNITAU - Universidade de Taubaté e Curso de Fisioterapia - USJT - Universidade São Judas Tadeu - E-mail: carolina.schmitt@ig.com.br

** Departamento de Epidemiologia - Faculdade de Saúde Pública / USP

*** Departamento Saúde Materno-Infantil - Faculdade de Saúde Pública / USP

Endereço: Av. Doutor Arnaldo, $7152^{\circ}$ andar sala 218 - São Paulo/SP - Cep: 01246-904
} 
e as doenças cardiovasculares ${ }^{4}$.

Estudos que investigam a tendência das causas de morte feminina no climatério são es$\operatorname{cassos}^{5,6,7}$ e em nosso meio, inexistentes. Certamente, o conhecimento das principais causa de morte no climatério poderá contribuir para a formulação de políticas de saúde pública de médio e longo prazo. Assim, objetiva-se caracterizar a tendência de mortalidade em mulheres de 35 a 64 anos no Brasil nos últimos anos.

\section{MÉTODO}

Foram coletados os dados de mortalidade do Sistema de Informações de Mortalidade do Datasus, Ministério da Saúde, no período de 1979 a 2004. ${ }^{8}$ Até o ano de 1995 as causas de morte foram registradas de acordo com a $9^{\text {a }}$. Revisão da Classificação Internacional das Doenças CID 9. ${ }^{9}$ A partir do ano de 1996, os dados foram registrados seguindo a $10^{\mathrm{a}}$. Revisão da Classificação Internacional das Doenças - CID $10 .^{10}$ Os óbitos foram considerados segundo o local de residência da falecida.

A tendência de mortalidade foi observada em relação aos dez primeiros capítulos mais freqüentes da CID como causas de morte e às décadas da faixa etária do climatério, de 35 a 44 , de 45 a 54 e de 55 a 64 anos $^{2}$ para o período. Foram calculados os coeficientes específicos de mortalidade por grupo etário e causas, baseado em 1000 indivíduos (número de mortes x 1000 / população estudada). Os dados da população de cada ano de estudo e cada década do climatério foram coletados das informações demográficas e socioeconômicas do Datasus ${ }^{11}$.

\section{RESULTADOS}

A população brasileira vem envelhecendo, tendendo à inversão da pirâmide populacional. Os censos de 1980 e de $2007^{12}$ mostraram que a população brasileira de 35 a 64 anos aumentou. Em 1980 o número total dessas mulheres residentes no Brasil foi de aproximadamente 13.300,000; em 1990, foi de 18.200,000; em 2000, foi de 25.100,000 e em 2007, foi de 27.900.

As dez primeiras causas de morte, segun- do os capítulos da CID 9 e 10 no Brasil, para as mulheres estudadas de 1979 a 2004 foram em ordem decrescente: doenças do aparelho circulatório, neoplasmas (neoplasias / tumores), sintomas, sinais e afecções mal definidas (sintomas, sinais e achados anormais de exame clínico e laboratorial), doenças do aparelho respiratório, causas externas (causas externas de morbidade e mortalidade), doenças do aparelho digestivo, doenças infecciosas e parasitárias (algumas doenças infecciosas e parasitárias), glândula endócrina, nutrição, metabólica (doenças endócrinas nutricionais e metabólicas), doenças do aparelho geniturinário e sistema nervoso e órgãos dos sentidos (doenças do sistema nervoso).

Em geral, houve destaque para três capítulos da CID no Brasil, conforme mostra a Figura 1: i) doenças do aparelho circulatório; ii) neoplasias e iii) causas mal definidas. As doenças do aparelho circulatório, apesar de serem as principais causas de morte, decresceram. Em contraposição, o coeficiente de mortalidade das neoplasias aumentou com o passar dos anos e as causas mal definidas tenderam a diminuir.

Ao analisar os coeficientes de mortalidade segundo cada década do climatério, todas as causas de morte aumentaram com o acréscimo de idade, com maior evidência para as neoplasias e doenças do aparelho circulatório. Os coeficientes para todas as causas foram menores que $1 / 1000$ para as mulheres de 35 a 44 anos, menor que 2/1000 mulheres para as de 45 a 54 anos e menor que 5/1000 mulheres para as de 55 a 64 anos. As demais causas de morte permaneceram com coeficientes abaixo de 1/1000 mulheres em todas as idades estudadas.

As regiões Sudeste, Sul e Centro-Oeste seguiram o mesmo padrão do Brasil da tendência de mortalidade. Entretanto, as circulatórias e mal definidas diminuíram, com maior ênfase para as circulatórias no Sudeste e Sul e as neoplasias tiveram discreta ascensão. Os coeficientes para as outras causas permaneceram constantemente baixos. O Centro-Oeste acompanhou esse padrão do Sul e Sudeste com menor proporção.

Entretanto, apesar da maioria das regiões acompanharem também o padrão do Brasil, o Norte e Nordeste tiveram suas peculiaridades. 
Figura 1: Coeficientes específicos de mortalidade das mulheres de 35 a 64 anos, segundo capítulos* da CID**. Brasil, 1979 a 2004.

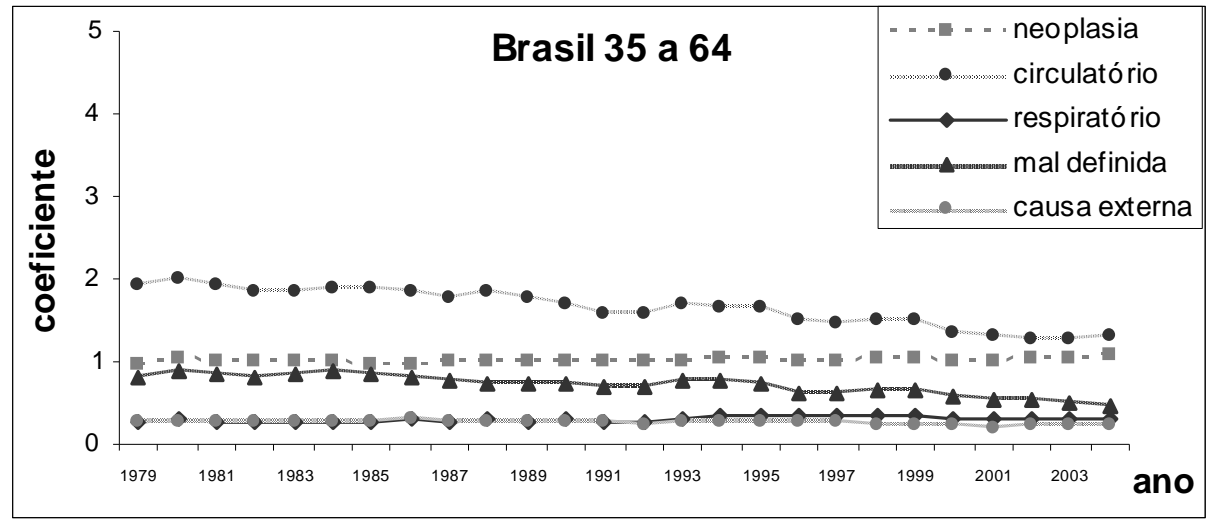

*Apresentação dos cinco primeiros capítulos mais freqüentes.

${ }^{* *}$ CID: Classificação Internacional de Doenças.

No Norte, os três primeiros capítulos de causa de morte apresentaram coeficientes próximos, em disposição de linearidade. Entre as mulheres de 35 a 65 anos as principais causas foram as doenças circulatórias, seguidas pelas mal definidas e neoplasias. Estratificando a idade, de 35 a 44 anos, as primeiras causas foram as mal definidas, seguidas ora pelas doenças circulatórias ora pelas neoplasias; de 45 a 64 anos, as circulatórias foram as mais freqüentes.

No Nordeste, as doenças mal definidas ocuparam a primeira posição, no entanto, decresceram a partir de 1984. As doenças do aparelho circulatório e neoplasias ocuparam a segunda e terceira posições, respectivamente, e aumentaram desde 1979.

\section{DISCUSSÃO}

Embora não seja possível converter o conjunto de dados da CID 9 para os dados da CID 10, pois a primeira tem 6.969 códigos enquanto que na segunda existem 12.420 códigos, notouse manutenção dos padrões antes e após 1995, o aumento ou o declínio das causas de morte no Brasil e regiões mantiveram-se os mesmos, não influenciando assim a tendência da mortalidade.

Apesar disso, é importante deixar claro que esta observação prévia pode ser uma limitação deste estudo, pois Janssen e Kunst ${ }^{13}$ (2004) concluíram que a comparação das revisões das CIDs pode levar a vieses de interpretação. Segundo Anderson et al. ${ }^{14}$ (2001), a comparação de dados entre a CID 9 e 10 baseada em uma única causa básica são mais complexas do que entre capítulos, já que mudanças introduzidas na CID 10 para as regras de seleção e modificação para chegar à causa básica têm impacto significante na seleção de certas condições, particularmente pneumonia e broncopneumonia.

O perfil epidemiológico da mortalidade no Brasil, com predomínio acentuado das doenças do aparelho circulatório e neoplasias como causas de morte, vinha crescendo desde 1930 até 1985 , quando este grupo superou as doenças infecciosas e parasitárias e passou a ser o principal responsável pelos óbitos do país. ${ }^{15}$

Esta tendência, especificamente em mulheres no climatério, mostrou ser diferente entre 1979 a 2004, diminuindo as mortes do aparelho circulatório, aumentando as neoplasias e mantendo as infecciosas, embora com maior freqüência das duas primeiras. Contudo, nunca é demais ressaltar a coexistência de mortes por doenças do atraso e da modernidade. Especialmente no Norte e no Nordeste, há predomínio das causas mal definidas e discreto aumento das doenças do aparelho circulatório e neoplasias. Em contraposição, 
nos EUA, as mortes por doenças do coração têm crescido de $30 \%$ para $33 \%$ e de câncer decrescido de $23 \%$ para $20 \%$ nos últimos anos. Os autores estimam para 2050 que as doenças do coração dominarão as causas de morte ${ }^{16}$.

Há poucos estudos sobre a mortalidade feminina e a influência do climatério. Dentre eles, destaca-se o de Hjartaker et al. ${ }^{5}$ (2005) que observaram uma coorte de 102.446 mulheres de 30 a 50 anos e encontraram que a mortalidade foi o dobro para mulheres com alto índice de massa corporal $\left(30 \mathrm{Kg} / \mathrm{m}^{2}\right)$, quando comparado com mulheres com índice normal, 18,5 a $24,9 \mathrm{Kg} / \mathrm{m}^{2}$. Após a menopausa, há tendência de acúmulo de gordura abdominal, o que pode contribuir para ocorrência de doenças circulatórias ${ }^{4}$. Neste trabalho, o coeficiente de mortalidade por estas doenças aumentou entre as mulheres com mais de 55 anos.

Ossewaarde et al. ${ }^{7}$ (2005) investigaram por dezessete anos a idade da menopausa, a causa de mortalidade e a expectativa de vida em 12.134 mulheres pós-menopausa. Os autores concluíram que mulheres com menopausa após 55 anos viveram dois anos a mais que as com menopausa antes dos 40 anos. Em particular, o risco de morte por doenças isquêmicas do coração foi $2 \%$ menor nas mulheres com menopausa tardia.

Corroborando com esses dados, Mondul et al. ${ }^{6}$ (2005) encontraram que a mortalidade por todas as causas teve uma taxa maior entre as mulheres que relataram a idade da menopausa de 40 a 44 anos e uma taxa menor, entre as de 50 a 54 anos. As principais causas de morte fo- ram doenças coronárias do coração, respiratórias, geniturinárias e causas externas. Neste trabalho, essas causas estavam entre as dez primeiras, todavia as três primeiras foram as doenças circulatórias, neoplasias e mal definidas. As circulatórias e mal definidas com tendência de declive e as neoplasias com aclive.

Monitorar a mortalidade em mulheres brasileiras no climatério é importante para facilitar as estratégias de políticas de saúde pública em médio e longo prazo já que, no Brasil, o papel da mulher tem mudado muito, especialmente nas últimas duas décadas. Isso se expressa na crescente participação feminina na força de trabalho e como chefe de família, nas mudanças de comportamento quanto ao controle da reprodução e na maior organização política, por meio de movimentos específicos de mulheres. Koop et al. ${ }^{17}$ (2004) apontaram que os estados de saúde, social e educacional da mulher de meia idade foram importantes preditores na mortalidade feminina e masculina. Apesar disso, o impacto dessas mudanças sobre a saúde ainda é pouco conhecido.

As três principais causas de morte no Brasil em mulheres de 35 a 64 anos foram decorrentes de doenças do aparelho circulatório, de neoplasias e sem causas definidas. Na população estudada, as regiões do Brasil seguem o mesmo padrão de causa de morte do país, com exceção do Norte e Nordeste. Houve uma tendência das doenças do aparelho circulatório e causas mal definidas decrescerem e as neoplasias aumentarem.

\begin{abstract}
There are few studies on women's mortality during climacteric, especially in Brazil. The objective of this study was to analyze the mortality trend among women between 35 and 64 years old in Brazil in the last years. Mortality data were collected in the Mortality Information System of Datasus, Ministry of Health, for the period 1979 to 2004. For the analysis, age- and cause-specific mortality rates were calculated for the ten more frequent chapters of the International Classification of Diseases as cause of death per decade of climacteric age group in the regions of Brazil. In Brazil, three chapters of the International Classification of Diseases predominated: cardiovascular diseases, malignant neoplasms, and ill-defined causes. The Southeast, South and Central West regions followed the same pattern of the country with regard to the position of the first three causes of death; however, cardiovascular diseases and ill-defined causes decreased while neoplasms increased. In the North region, although the same causes presented similar rates, cardiovascular diseases prevailed in most of the study period, but ill-defined causes were more frequent than neoplasms. In the Northeast, the main causes were the ill-defined ones, although they declined from 1979 to 2004. Cardiovascular diseases and neoplasms were in the second and third positions, respectively, and increased in the study period.
\end{abstract}

Keywords: Mortality; women's health; epidemiology. 


\section{REFERÊNCIAS}

1. WHO. Statistical Information System. WHOSIS. Core Health Indicators from the latest World Health Report. Geneva; 2006. [acesso em 21 abril 2006]. Disponível em: http://www3.who.int/ whosis/core/core_select.htm.

2. Notelovitz M. Climateric medicine and science: a societal need. In: Notelovitz M. The Climateric in Perspective. Lancaster: M.T.P. Press; 1984. p. 1921.

3. Aldrighi JM, Calvoso Junior R, Faludi AA, Mansur AP. Climatério e menopausa. In: ALDRIGHI JM. Doença Cardiovascular no Climatério. São Paulo: Atheneu, 2005. p. 23-30.

4. Aldrighi JM, Aldrighi CMS, Aldrighi APS. Alterações sistêmicas no climatério. Rev Bras Med 2002; 59:15-21.

5. Hjartaker A, Adami HO, Lund E, Weiderpass E Body mass index and mortality in a prospectively studied cohort of Scandinavian women: the women's lifestyle and health cohort study. Eur J Epidemiol 2005; 20(9):747-54.

6. Mondul AM, Rodriguez C, Jacobs EJ, Calle EE Age at natural menopause and cause-specific mortality. AM J Epidemiol 2005; 162(11):1089-97.

7. Ossewaarde ME, Bots MI, Verbeek AL, Peeters PH, van der Graaf Y, Grobbee DE et al. Age at menopause, cause-specific mortality and total life expectancy. Epidemiology 2006; 16(4):556-62.

8. Datasus. Informações de Saúde. Demográficas e socioeconomicas. Brasil; 2006. [acesso em 21 abril 2006]. Disponível em: http://www.datasus.gov.br/.

9. OMS. Organização Mundial de Saúde - Manual da Classificação Internacional de Doenças, Lesões e Causas de Óbitos. $9^{a}$ Revisão. 1975. São Paulo: Centro da OMS para Classificação das Doenças em Português, 1978.

10. OMS. Organização Mundial de Saúde Classificação Estatística Internacional de Doenças e Problemas relacionados à saúde: Classificação Internacional de Doenças-10. São Paulo: EDUSP, 1995.

11. Datasus. Informações de Saúde. Estatísticas vitais, mortalidade e nascidos vivos. Brasil; 2006. [acesso em 21 abril 2006]. Disponível em: http:// www.datasus.gov.br/.

12. IBGE. Censo Demográfico. Brasil; 2008. [acesso em 21 fev 2008]. Disponível em: http:// www.ibge.gov.br/.

13. Janssen F, Kunst N. ICD coding change and discontinuities in trends in cause-specific mortality in six European countries, 1950-99. Bull of World Health Organ 2005; 82(12):904-913.

14. Anserson RN, Minino AM, Hoyert DL, Rosenberg HM. Comparability of cause of death between ICD 9 and ICD 10: preliminary estimates. Natl Vital Stat Rep. 2001; 49:1-32.

15. Prata PR. A transição epidemiológica no Brasil. Cad Saude Publ 1992; 8(2):168-175.

16. Sonnenschein E; Brody JA. Effect of population aging on proportionate mortality from heart disease and cancer. J Gerontol B Psychol Sci Soc Sci 2005; 60(2):S110-112.

17. Kopp MS, Skrabski A, Kawachi I, Adler NE. Low socioeconomic status of the opposite sex is a risk factor for middle aged mortality. J Epidemiol Community Health 2005; 59:675-678. 